\title{
Le Roman d'un Nègre à la recherche d'un titre, suivi d'un post-scriptum, dix ans après
}

\section{Roger Little}

\section{(2penEdition}

\section{Journals}

Édition électronique

URL : https://journals.openedition.org/coma/6983

DOI : $10.4000 /$ coma.6983

ISSN : 2275-1742

Éditeur

Institut des textes \& manuscrits modernes (ITEM)

\section{Référence électronique}

Roger Little, «Le Roman d'un Nègre à la recherche d'un titre, suivi d'un post-scriptum, dix ans après », Continents manuscrits [En ligne], 17 | 2021, mis en ligne le 15 octobre 2021, consulté le 08 janvier 2022 URL : http://journals.openedition.org/coma/6983; DOI : https://doi.org/10.4000/coma.6983

Ce document a été généré automatiquement le 8 janvier 2022.

\section{(C) $\odot \Theta \Theta$}

Continents manuscrits - Génétique des textes littéraires - Afrique, Caraîbe, dispora est mis à disposition selon les termes de la licence Creative Commons Attribution - Pas d'Utilisation

Commerciale - Pas de Modification 4.0 International. 


\title{
Le Roman d'un Nègre à la recherche d'un titre, suivi d'un post-scriptum, dix ans après
}

\author{
Roger Little
}

\section{NOTE DE L'ÉDITEUR}

Les coordinateurs du dossier ont prié Roger Little, ancien titulaire de la chaire de français au Trinity College Dublin et spécialiste de René Maran, de bien vouloir se prêter ci-dessous à une actualisation de son étude importante de 2013, «Le Roman d'un nègre à la recherche d'un titre ", originellement publiée dans la revue Présence africaine, $\mathrm{n}^{\circ} 187-188\left(1^{\mathrm{er}}\right.$ et $2^{\mathrm{e}}$ semestres 2013) dans un dossier qui reprend des textes présentés au colloque parisien de décembre 2010 consacré à René Maran. Ils lui sont très reconnaissants d'avoir accepté cette proposition. Roger Little tient pour sa part à remercier les responsables de la revue d'avoir autorisé cette reprise. Sa gratitude est grande aussi envers $\mathrm{M}^{\text {mes }}$ Roseline Garcia Ballester, Laura Gauthier-Blasi, Tina Harpin et Claire Riffard, et MM. Bernard Michel et Charles Scheel.

1 Le titre du Journal sans date de René Maran, publié en 1927, devenu Un homme pareil aux autres dans sa version définitive de 1947 , s'est avéré particulièrement instable ${ }^{1}$. Ce n'est pas un texte qui a beaucoup retenu l'attention des critiques, mais ce n'est pas du texte qu'il s'agit ici en l'occurrence, malgré son intérêt pour ceux, d'une part, qui se penchent sur l'autobiographie intime de l'auteur à la suite du succès de Batouala ${ }^{2}$ et pour ceux, d'autre part, qui s'attachent à l'analyse sociologique ou littéraire des relations et mariages interraciaux - mixtes comme on dit maintenant, sans préciser de quel mélange il s'agit. En l'occurrence il est question du seul titre et ses différents avatars.

Outre les deux titres qu'on lui connait, le roman s'est vu attribuer plusieurs titres de travail provisoires. René Maran aurait commencé à parler d'un Roman d'un nègre dès la 
fin de l'année 1921, par exemple dans sa correspondance avec Charles Barailley ${ }^{3}$, et il en est passagèrement question dans un article de Jean-Michel Renaitour dans La Pensée française du 14 janvier $1922^{4}$. Dans une lettre à son ami éditeur Léon Bocquet, datée du 18 juin 1924, il évoque la parution prochaine du Roman d'un nègre ${ }^{5}$, et ce titre est également annoncé dans Le Petit Roi de Chimérie qui paraît en $1924^{6}$. Un extrait intitulé "Retour en France " paraît en effet sous le titre général Le Roman d'un nègre dans le périodique Je sais tout le 15 novembre de cette année ${ }^{7}$, précédé d'une photo de « femmes à plateau » du Moyen-Chari et d'un « chapeau » qui commence comme suit :

Une blanche peut-elle épouser un noir? Posée, voici quelques années, par un hebdomadaire illustre ${ }^{8}$, cette question fournit - chez les blanches Parisiennes - le sujet d'une enquête passionnément discutée. Il faut entendre maintenant la voix de la contre-partie : celle des « hommes de couleur». M. René Maran est l'un d'eux. Il appartient à l'élite intellectuelle noire, et même à l'élite intellectuelle tout court, puisqu'il fut lauréat de l'enviable Prix Goncourt avec Batouala. - Sous ce titre : Le Roman d'un Nègre, il va raconter l'histoire d'un fonctionnaire colonial noir, marié avec une blanche.

3 Félix Éboué écrit à Maran un an plus tard, le 9 novembre 1925: «j'attends avec impatience le roman d'un nègre.» Mais de poursuivre: «Je n'aime pas ce titre, qui pourrait être un sous-titre, et encore, mais je suis persuadé que ce sera très bien ${ }^{9}$.» $\mathrm{Ce}$ conseil amical ${ }^{10}$ serait-il un motif suffisant pour abandonner un titre en effet bateau et banal (le « et encore » d'Éboué est parlant), pour ne pas dire franchement mauvais ? Qui sait? Quoi qu'il en soit, c'est sous le titre Journal sans date que ce roman à la recherche d'un titre paraitra en avril 1927, même si, dans une lettre que Maran envoie à Alain Locke le 4 mars 1927, l'auteur semble s'en excuser d'avance: «Je pense pouvoir vous adresser, en avril, un exemplaire de mon Djouma, et un exemplaire de la revue où paraîtra, sous sa forme nouvelle et définitive, Le Roman d'un Nègre, qui sera intitulé, pour la circonstance, Journal sans date ${ }^{11}$. » En juillet 1927 toutefois, on fait allusion, dans La Pensée française, à un récit intitulé Jean Veneuse, du nom du personnage principal du roman $^{12}$. Confusion sur confusion ${ }^{13}$ ! Mais la ronde des variantes du titre ne s'arrête pas là. En 1932, dans Les Feuillets littéraires, paraît une version courte du roman sous le titre Défense d'aimer ${ }^{14}$.

Le titre Journal sans date est-il plus heureux que Roman d'un nègre? Il a le mérite du moins, tout comme Défense d'aimer, de rendre universelle la question du «couple mixte ", dont les enjeux ne sont nullement limités en effet au mariage entre un Noir et une Européenne. Maran savait-il qu'il existait dans la littérature française, depuis le dix-huitième siècle, de nombreux romans traitant du «couple domino » Blanche et Noir ${ }^{15}$ ? Il ne pouvait ignorer l'Othello de Shakespeare - il en fait même le titre d'un de ses poèmes ${ }^{16}$-, mais il ne serait ni le premier ni le dernier romancier français à ignorer les récits qui traitent du couple interracial et dont les auteurs croient très souvent explorer des terres entièrement inconnues. En l'occurrence, il serait traditionnellement question d'un double affranchissement, celui de l'homme noir, qui rejoint en quelque sorte le camp de ses maîtres, répondant à celui de la femme blanche qui, elle, se libère de la tutelle des hommes. C'est donc une aventure passionnante que l'étude de ce phénomène littéraire d'une double décolonisation... à laquelle nous devons pourtant renoncer ici, tout comme nous renonçons par conséquent à revenir sur la critique malveillante et mal conçue que formule Frantz Fanon à l'égard du $\operatorname{roman}^{17}$. 
Revenons donc aux nombreuses hésitations de Maran sur le titre de son roman et à son choix définitif. Les traces détaillées de son élaboration nous manquent, et trois rapports de lecteurs sur le manuscrit du Roman d'un Nègre, ni datés, ni signés, faits pour la maison d'édition Albin Michel qui, nous l'avons vu, rééditera en 1962, et donc de manière posthume, Un homme pareil aux autres, n'y font pas allusion ${ }^{18}$. De deux choses l'une : soit, dans les années quarante, l'éditeur Arc-en-ciel a soufflé à René Maran que son titre Roman d'un Nègre ne convenait vraiment pas, soit de son propre chef l'auteur a pris l'initiative d'en choisir un autre. Il est peu probable que l'éditeur ait imposé un nouveau titre, mais si c'était le cas, mon hypothèse serait nulle et non avenue. Nous sommes toutefois en droit, me semble-t-il, de supposer que, dans un cas comme dans l'autre, c'est Maran qui aurait décidé de la version définitive, ou qui l'aurait du moins entérinée, mais il est curieux de constater que malgré la publication sous le titre définitif en 1947, Maran soumet peu avant sa mort un manuscrit (remanié ?) toujours intitulé Le Roman d'un Nègre et que c'est toujours ainsi qu'il évoque son ouvrage.

6 Mon hypothèse porte sur ce choix final. Certes l'expression figure dans le texte du roman : « je ne sais qu'une chose : c'est que le nègre est un homme pareil aux autres, et que son cœur, qui ne paraît simple qu'aux ignorants, est aussi compliqué que peut l'être

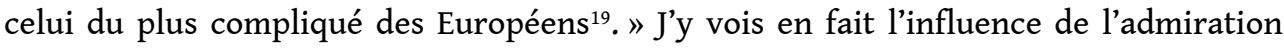
qu'avait Maran pour le roman d'Émile Nolly, pseudonyme du capitaine Émile Détanger (1880-1914) : Hiên le Maboul. Pourquoi évoquer une source en apparence si obscure, si éloignée géographiquement de l'expérience vécue de Maran ? C'est que dans sa « Lettre ouverte au professeur Alain Locke de l'Université d'Howard (États-Unis)», publiée le 15 juin 1924 dans la troisième livraison du journal qu'il dirigeait avec Kojo Tovalou Houénou, Les Continents, il écrit : "Donnez-vous la peine de lire, cher Monsieur Locke, Des Inconnus chez moi, de Mme Lucie Cousturier; Hiên le Maboul, de feu le capitaine d'infanterie coloniale Détanger, en littérature Émile Nolly; La Randonnée de Samba Diouf, des frères Tharaud; certains passages extraits des Thibaut [sic], de Roger Martin $\mathrm{du}$ Gard, et quelques récits africains de Jean-Richard Bloch ${ }^{20}$. » C'est qu'il revient aussi sur certains des mêmes ouvrages dans sa préface de 1957 à une nouvelle édition de Des inconnus chez moi, de Lucie Cousturier, où il loue la manière dont elle, tout comme Émile Nolly dans son roman indochinois, et les frères Tharaud dans La Randonnée de Samba Diouf, dépeint «ce qu'on était convenu d'appeler par antiphrase, aux colonies, des “engagés volontaires" $»^{21}$.

\section{Hiên le Maboul, texte catalyseur}

7 Hiên le Maboul parut d'abord en feuilletons dans La Revue de Paris en octobrenovembre 1908 pour être publié peu de temps après ${ }^{22}$. Situé en Indochine française, il retrace les heurs et, surtout, les malheurs du tirailleur annamite éponyme. Recruté par hasard et par force, le bûcheron simplet a la chance de rencontrer un lieutenant exceptionnellement compréhensif connu comme "l'Aïeul à deux galons", dont le tempérament et les fonctions spécialisées de cartographe lui permettent de prendre ses distances physiques et morales de la chaîne d'autorité militaire normale.

Tout au long du roman, on peut suivre le fil conducteur qui, malgré tout, et malgré surtout son inaptitude initiale, fait de Hiên un homme pareil aux autres. Pour les besoins de la cause, et parce que je ne peux pas supposer que les lecteurs de Maran 
aient nécessairement lu le roman de Détanger, je me trouve dans l'obligation de citer longuement les principaux passages qui confortent mon hypothèse. Au début :

Il vécut ainsi, chaque jour moins sociable et plus proche de la nature, chaque jour plus sauvage et moins pareil aux autres hommes. À vingt ans, il fut une sorte de géant maigre aux yeux égarés, à la chevelure inculte, aux gestes maladroits ; et l'opinion se confirmait qu'il était fou $(20 / 9)^{23}$.

"L'Aïeul à deux galons » se charge du mieux qu'il peut de son éducation dans les voies et façons du monde :

Je lui enseignerai la vie: il saura qu'un homme en vaut un autre [...]. Il connaittra, quelque jour, que la valeur des gens se mesure à l'opinion qu'ils ont d'eux-mêmes ; il verra que l'abîme qui sépare de lui le reste de l'humanité n'est qu'un ruisseau; une fois apprise la douzaine de grimaces indispensables à notre existence quotidienne, il sera un homme comme toi et moi. (83-84/49)

En dépit de nombreuses épreuves, le lieutenant pourra juger beaucoup plus loin que le processus est achevé : «À cette heure, son éducation d'homme pareil aux autres hommes était achevée, puisqu'il percevait maintenant le sanglot infini de l'humanité »(242/145). Mais il est loin du compte : Hiên est frustré dans ses efforts d'exprimer les douloureuses émotions du dépit amoureux, dépit doublé d'un mépris délibéré de la part de sa fiancée, Maÿ :

Un instant, il [Hiên] eut l'étrange désir de se rouler dans la poussière, de hurler, comme se roulent et comme hurlent, pour se soulager, les bêtes blessées. Mais il était un homme civilisé, un homme pareil aux autres hommes, et rien ne sortit de sa gorge serrée. (258/155)

C'est seulement vers «l'Aïeul à deux galons" qu'il peut se retourner pour une éventuelle consolation :

- [...] Souffle sur ma douleur : elle s'envolera de mon cœur où elle a fait son nid. [...] tu as porté la lumière dans mon âme obscure d'enfant des bois...

- J'ai eu tort, trois fois tort! confesse l'Aïeul ; j'aurais dû laisser ton âme à sa pénombre, à son heureuse inconscience. Tu avais le bonheur, ne connaissant de l'humanité que les gestes animaux. [...] Mais quoi ! tu m'as supplié, tu m'as dit : «Je veux être un homme comme les autres hommes et je saurai me faire aimer de Mä̈... » Je t'ai instruit, je t'ai appris les grimaces essentielles, je t'ai révélé tes semblables. [...] Tu as appris à vivre. La suprême leçon, celle qui ne pouvait te venir de moi, la vie s'est chargée de te la donner : elle t'a fait connaitre la désillusion et la douleur.

[...]

- Ainsi mes prévisions se sont réalisées : tes illusions sont mortes, et te voilà, tombé de ton rêve et pleurant pitoyablement... [...] Lorsque tes larmes auront séché, tu seras certain que ton éducation est parachevée et que tu es un homme, puisque tu as connu la douleur. (271-272/162-163)

Le chapitre qui montre le suicide de Hiên par pendaison s'achève sur la phrase suivante, qui répète et clôt le motif que j'ai voulu souligner ici et qui, à mon sens, suggéra à Maran le titre définitif de son roman partiellement autobiographique : « Ainsi finit Hiên le Maboul qui voulut vivre comme les autres hommes » (276/166).

11 Une telle hypothèse en appelle de toute évidence d'autres qui en découlent, que ce soit la douloureuse idée que Maran avait de lui-même comme un grand solitaire incompris, un étranger dans ce monde malgré ses réussites apparentes ${ }^{24}$. Beaucoup pourraient lui envier la sécurité de sa fonction coloniale, sa lumineuse intelligence assise sur une éducation solide - quelle chance d'avoir eu le grand érudit Fortunat Strowski comme professeur de lettres et ce M. Lambinet, André, à qui il rendra un si vibrant hommage ("Vous n'avez voulu être qu'un homme comme les autres ${ }^{25} »$ ), comme professeur de latin-, son extrême sensibilité humaine doublée de scrupules non moins extrêmes, 
l'immense succès, même contesté, de son prix Goncourt de 1921. Mais il y a les souffrances du revers de la médaille: cette jalousie tenace dont il est victime, empreinte notamment du mépris du racisme. On a bien l'impression que la sympathie, l'empathie même, qu'il ressent pour la représentation d'Hiên par Émile Nolly provient $\mathrm{du}$ fait qu'elle résumerait ses propres expériences d'injustice dans la situation coloniale et trouverait un écho profond dans Jean Veneuse, héros de son roman. Si l'on veut bien admettre mon hypothèse, une autre conclusion s'impose : le rattachement de Maran, dans la réalité comme chez ses critiques, au seul monde noir - Afrique et AntillesGuyane - aurait tendance à négliger sa position de principe contre non seulement les abus de la colonisation mais encore contre la déshumanisation du colonialisme en tant que système. Lui-même aurait évolué dans ce sens. Le 19 août 1921, il pouvait encore écrire à Jean-Michel Renaitour :

[...] tout en prévoyant d'innombrables moqueries, je voudrais intéresser le peuple de France, si riche en esprit généreux, à la question noire. On ne sait peut-être pas assez l'évolution de cette race, ni la marche ascendante de sa haine contre tous les peuples de proie qui l'ont brimée, ou la briment encore, moralement lorsque ce n'est pas physiquement. Il n'y a pas là incompatibilité, mais fureur de fauve blessé, qui recouvre ses forces pour assaillir son meurtrier. Il faut excuser qui se venge d'avoir été malheureux ${ }^{26}$.

Le roman d'Émile Nolly aurait servi de catalyseur en lui ouvrant les yeux à une région du monde qu'il ne connaissait pas et en faisant ainsi valoir le principe au-delà de l'exemple. Le titre même d'Hiên le Maboul renvoie déjà à plusieurs régions du monde : l'Indochine dans le nom du personnage principal, bien sûr, mais aussi l'Afrique des grandes savanes par le biais de la hyène dont il fait l'écho, le Maghreb enfin, vu l'origine et la consonance arabes du mot maboul. L'impact sur l'esprit de René Maran aurait été d'autant plus fort, plus lourd de conséquences et de résonances que si la scène avait été l'Afrique tropicale qu'il connaissait si bien. «Car mon Jean Veneuse, dont j'ai fait un nègre, écrit-il dans une lettre à son amie Daphné Trevor le 27 octobre 1947, j'aurais pu le peindre tout aussi bien sous les traits d'un juif ou d'un Indochinois [sic ${ }^{27}$. » En fin de compte, René Maran lui-même ne souhaitait-il pas du fond de son cœur et du haut de son esprit être considéré par tous comme un homme pareil aux autres?

L'avant-propos (tout en italique) d'Un homme pareil aux autres se termine sur des remarques qui, en allant au cœur de son intérêt profond, nous incitent à repenser les approches critiques empruntées jusqu'ici. Il n'y est nullement question, notamment, d'autobiographie : ce n'est pas là son propos. C'est le roman, simple au fond, et rédigé d'une main de maître, d'un amour qui fait fi des préjugés raciaux, voire racistes, et qui nous invite encore à questionner nos préventions à ce sujet. Aussi s'inscrit-il dans le droit fil de son œuvre romanesque et de ses préoccupations humaines :

Le livre que voici n'est, au fond, que le voyage d'une race à une autre et d'un cœur à un autre cœur. Puisse-t-il, par sa simplicité sans apprêt et son dépouillement, imposer silence aux préjugés, aux sophismes et aux partis pris, qui cherchent à transformer les arrêts changeants de sciences trop souvent faillibles en autant d'impératifs ethniques ${ }^{28}$.

Ce qui était vrai en 1927, en 1947 et encore en 1962, requiert toujours notre attention. 


\section{Post-scriptum, dix ans après}

Mon regard en arrière révèle non Eurydice mais bien M. Jourdain, découvrant en l'occurrence non que j'écrivisse en prose, mais que je faisais, d'une certaine manière, de la critique génétique. La préparation d'une édition critique génétique de l'œuvre de René Maran par une équipe de l'ITEM constituée sous l'autorité de Claire Riffard, m'a donc plongé dans un travail de longue haleine où je devais rapidement apprendre les bases de cette science nouvelle pour moi.

Sous la tutelle de cette équipe, un groupe s'est formé qui se penche sur l'évolution du roman qui deviendra en définitive Un homme pareil aux autres (Albin Michel, 1962, après avoir été publié sous ce titre par les éditions Arc-en-ciel en 1947). La découverte de documents qui n'étaient pas disponibles il y a dix ans permet à celles qui, bien plus que moi - Laura Gauthier-Blasi et Tina Harpin -, établissent les variantes, de tenter de résoudre les difficiles problèmes de chronologie posés par des manuscrits (gracieusement déposés par les ayants droit qui en ont autorisé la saisie) souvent non datés. Il s'agit aussi de combler certaines lacunes d'information que révèlent ces documents en se référant aux correspondances inédites de l'auteur. Mais il reste bien des conjectures où manquent les preuves qui permettraient des conclusions définitives.

L'un des documents récemment mis à la disposition de l'équipe provient du fonds Maran de l'Université de Dakar où il a été déposé par la veuve de l'auteur. Classé Ms 15, il porte le titre, barré en l'occurrence : " UN NÈGRE PARLE », remplacé en dessous par «Un Homme comme un autre » suivi de la mention : « récit » (voir fig. 1). Non daté, il serait quand même antérieur à un autre manuscrit du roman, également non daté, mis à la disposition de l'équipe par Bernard Michel, dont René Maran était le grand-père. $\mathrm{Ce}$ manuscrit modifie encore les titre et sous-titre : « Un homme comme les autres :

Roman » (voir fig. 2). On se perd en hypothèses. On peut toutefois, sous toute réserve, proposer une date pour le manuscrit de Dakar, car René Maran écrit à Manoel Gahisto le 15 octobre 1939, lors d'un de ses séjours à Paris : « Le Roman d'un Nègre, qui devait paraître ce mois-ci, sous le titre Un Nègre Parle, ne paraîtra que lorsque Hitler ne pourra plus mordre ». Cette mention, sur laquelle Maran revient dans une lettre adressée à l'éditeur Sorlat le 5 juin $1944^{29}$, paraît nous autoriser à situer le manuscrit de Dakar aux alentours de 1939. Aurait-il servi de base pour l'édition Arc-en-ciel de 1947, ou pour celle, en l'occurrence posthume, chez Albin Michel, de 1962 ? Ce qui est clair, c'est que le titre définitif est le fruit d'une très longue hésitation enclenchée au moins un quart de siècle plus tôt. 
Fig. 1 : Page de titre : «UN NĖGRE PARLE / Un Homme comme un autre : récit ».

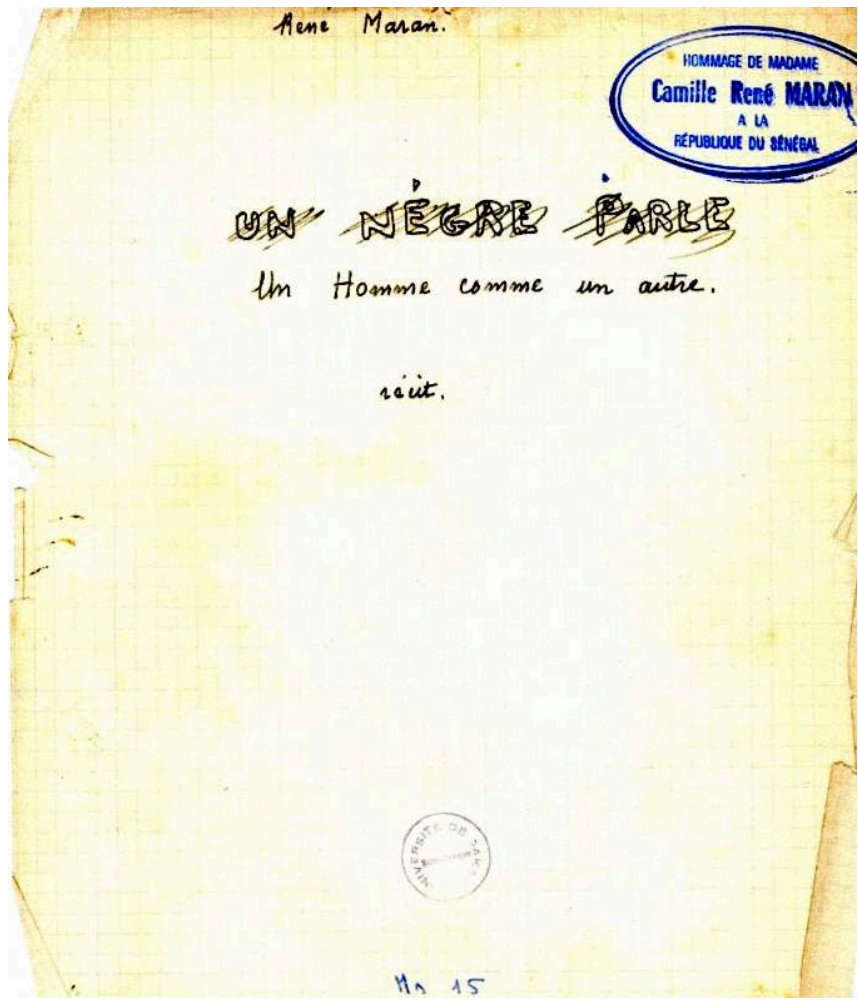

(c) Fonds Maran, Université Cheikh Anta Diop, Dakar, Ms 15

Fig. 2 : Page de titre : « Un homme comme les autres : roman ».

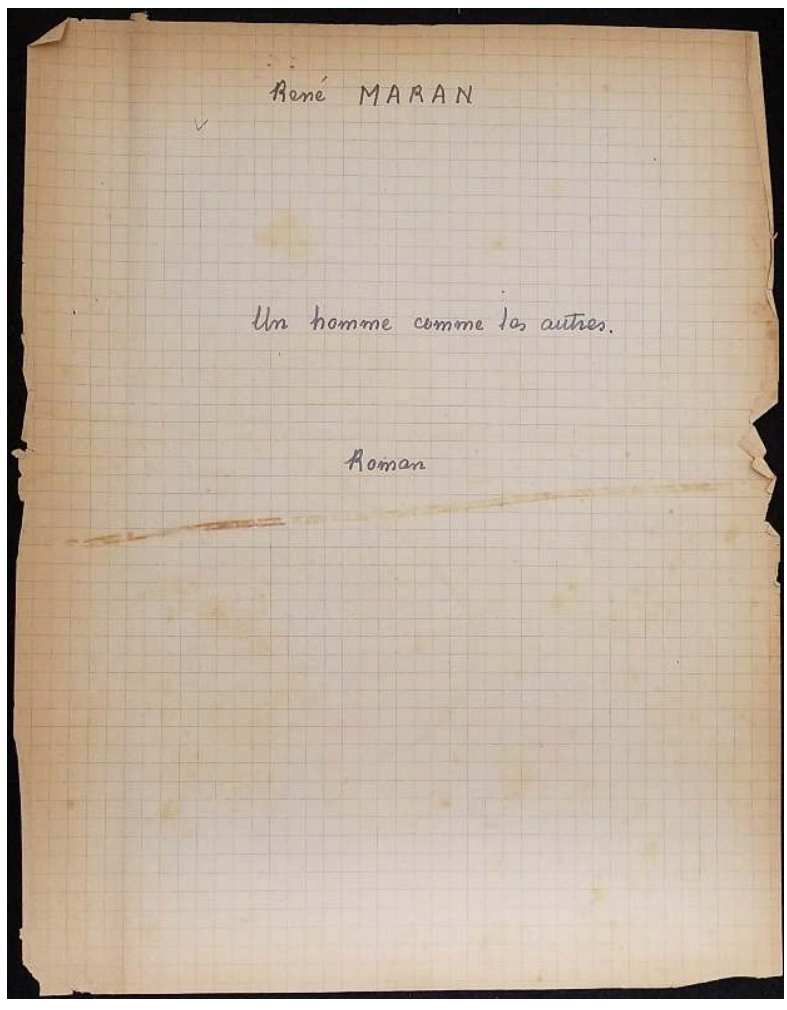

(c) Fonds Bernard Michel 
17 Parmi les «ouvrages du même auteur en préparation » indiqués dans la première édition de Batouala figure "Le Roman d'un Nègre, roman ». Ce doit être la première mention publique de ce titre : le directeur de la maison Albin Michel annonce à Manoel Gahisto, dans une lettre dactylographiée du 25 juillet 1921: " "BATOUALA" est en vente ${ }^{30} »$.

Une copie dactylographiée de l'abondante correspondance échangée entre l'écrivain et traducteur Manoel Gahisto (1878-1948) et René Maran m'a été aimablement remise par Bernard Michel, petit-fils de ce dernier. Elle est révélatrice à plus d'un titre. Maran fait mention dans une lettre datée du 17 octobre 1913, de Fort Sibut en Oubangui-Chari, de plusieurs projets en cours dont un porte le titre « Une amie ». C'est un « récit en termes et nuances psychologiques, sorte de contribution que j'essaie d'apporter à l'amitié amoureuse. Je me suis quelque peu autobiographé en ce manuscrit que je revois infiniment et corrige - ainsi que toutes mes productions ». Le 26 novembre de la même année, il écrit : «J'ai revu et mis à nouveau sur l'établi des chapitres de Batouala le M. et de Une amie. Bref, je n'ai pas perdu mon temps. » " Une amie » serait-ce une première ébauche de ce qui sera à la fin Un homme pareil aux autres? On ne le saura peut-être jamais, car le 10 avril 1914 Maran paraît en avoir interrompu la rédaction : " "Batouala de makondji" [sic] ${ }^{31}$, “Une amie" n'avancent plus, depuis longtemps. De loin en loin, je vous dis que je les revois. C'est vrai sans l'être. Tous les chapitres sont esquissés, j'essaie de m'inviter au travail. Malheureusement, le travail me dégoûte. » Dès le 5 décembre 1920, Maran écrit de Bordeaux : «En tous les cas j'ai arrêté le titre définitif du roman qui succédera à cet ouvrage [Le Petit Roi de Chimérie]. Le voici : "Le Roman d'un Nègre". Ce sera, vous le devinez bien, un roman à thèse, où il y aura des notes autobiographiques. »

L'on suit de loin en loin l'évolution de l'écriture. Dans une lettre du dernier jour de l'année, on lit: " "Le Roman d'un Nègre" atteint sa vingtième page. » Puis, à bord du Tchad qui l'emmène vers l'Oubangui-Chari, 17 février 1921, il note: "depuis mon départ, j'ai poussé la première partie du "roman d'un Nègre" ": "Couple mixte, c'est-àdire : mari nègre, - martiniquais comme moi, paraît-il, - femme blanche, - parisienne, et ancienne dactylographe au Ministère de la guerre." De Fort-Archambault, le 21 juillet 1921, il confie à Gahisto :

«Le Roman d'un Nègre » bouillonne dans mon esprit. Si je l'y laisse, parce que je n'ai pas de loisirs, trop longtemps encore, ce bouillon deviendra un concentré âpre et dur. Vous m'aviez, jadis, conseillé d'écrire: «Au pays des Blanches». Je commence à avoir peur que ce livre, qui sera par moment un peu autobiographique, ne devienne cela. Mais qu'importe! Roman à thèse, où seront opposées deux races, et prouvé que le désir est l'ennemi du désir, l'homme ennemi de l'homme et de la femme aussi.

19 À aucun moment, Maran ne semble avoir retenu « Au pays des Blanches » comme titre. Enfin, de Koumra, le 9 août 1922, il écrit : « Suis trop fatigué pour travailler au "roman d'un Nègre". »

Me limitant pour les besoins de la cause au seul titre, un exemple révélé par l'un des documents nouvellement accessibles est révélateur du problème auquel nous faisons face. La toute première page du tapuscrit (utilisant un ruban bleu et numéroté III en haut à droite) que René Maran aurait remis en 1927 pour publication dans les Euvres libres comporte, outre des annotations (en rouge) de la personne responsable de la préparation $\mathrm{du}$ texte pour la revue, une intervention de l'auteur. Le titre 
dactylographié : $L E R O M \cap N D^{\prime} U N N E G R E$, ainsi espacé, est barré. Au-dessus, Maran a écrit de sa main : Journal sans Date (voir fig. 3).

Fig. 3 : Page de titre : «LE ROMAN D’UN NEGRE / Journal sans Date », 1927.

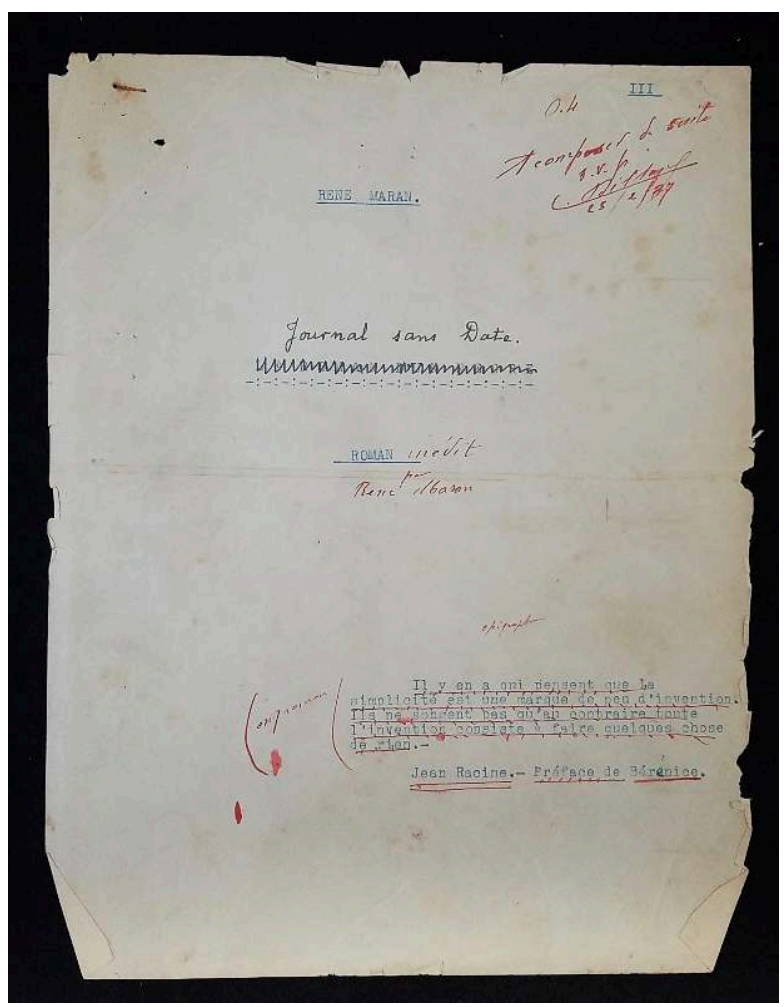

(c) Fonds Bernard Michel

21 Qu'est-ce qui a motivé ce changement, qui paraît être de dernière heure ? Nous avons émis certaines hypothèses dans notre étude reproduite ci-dessus : conscience d'un titre banal (mais Journal sans date l'est-il moins ?), intervention de Félix Éboué ? On pourrait y ajouter celle d'une suggestion faite par les rédacteurs des Euvres libres. Comment trancher en l'absence d'autres informations? Terminer ainsi par un point d'interrogation paraît être en l'occurrence la seule conclusion responsable, définitivement provisoire.

\section{BIBLIOGRAPHIE}

\section{Archives}

Correspondance Alain Locke - René Maran, archives de Howard University, Washington D.C.

Correspondance René Maran - Manoel Gahisto, archives privées

Fonds René Maran, Université Cheikh Anta Diop, Dakar 
Fonds Bernard Michel, succession René Maran

Publications de René Maran

MARAN, René, Batouala : véritable roman nègre, édition définitive, Paris, Albin Michel, 1938

(impression de 1975)

MARAN, René, Le Cour serré, Paris, Albin Michel, 1931

MARAN, René, Défense d'aimer, Les Feuillets littéraires, Paris, Fayard, $n^{\circ} 4$ (1932)

MARAN, René, Félix Éboué, grand commis et loyal serviteur (1884-1944) [1957], rééd. prés. Bernard

Mouralis, coll. « Autrement Mêmes », n 37, Paris, L’Harmattan, 2007

MARAN, René, Journal sans date, dans Euvres libres, Paris, Fayard, 1927, p. 105-236

MARAN, René, Le Livre du souvenir : poèmes, 1909-1957, Paris, Présence Africaine, 1958

MARAN, René, «Othello », La Revue du monde noir, n 3, (janvier 1932), p. 33

MARAN, René, Le Petit Roi de Chimérie, Paris, Albin Michel, 1924, avec une importante préface de Léon Bocquet

MARAN, René, « Préface » à la réédition de 1957 de Lucie Cousturier, Des inconnus chez moi [1920], repr. p. XxIX-XXX dans rééd. prés. Roger Little, Paris, L'Harmattan, coll. « Autrement Mêmes », $n^{\circ} 1,2001$

MARAN, René, Un homme pareil aux autres, Paris, Arc en Ciel, 1947

MARAN, René, Un homme pareil aux autres, nouvelle édition, Paris, Albin Michel, 1962

MARAN, René, La Vie intérieure : poèmes (1909-1912), Paris, Le Beffroi, 1912

\section{Ouvrages cités}

BLOCH, Jean-Richard, À la découverte du monde connu, Paris, Éditions de la NRF, 1924

COUSTURIER, Lucie, Des inconnus chez moi [1920], rééd. prés. Roger Little, Paris, L'Harmattan, coll.

« Autrement Mêmes », nº 1, 2001

FANON, Frantz, Peau noire, masques blancs, Paris, Seuil, 1952 (souvent réédité)

GENESTE, Elsa, « Autour du Batouala de René Maran : réflexions sur quelques formulations racistes et antiracistes du mot "nègre" ", Nuevo Mundo/Nuevos Mundos, Paris, EHESS, 2010 : http:// nuevomtmdo.revues. org/60301

LITTLE, Roger, Between Totem and Taboo: Black Man, White Woman in Francographic Literature, Exeter, University of Exeter Press, 2001

LITTLE, Roger, « Escaping Othello's Shadow: Un homme pareil aux autres, Ô pays, mon beau peuple! and Un chant écarlate », ASCALF Yearbook [ISSN 13653938], 1 (1996), p. 94-112

LITTLE, Roger, « Le Roman d'un nègre à la recherche d'un titre », Présence africaine, $\mathrm{n}^{\circ} 187-188$ ( $1^{\mathrm{er}}$ et $2^{\mathrm{e}}$ semestres 2013), p. 167-174

NOLLY, Émile, pseud. d'Émile Détanger, Hiên le maboul, Paris, Calmann-Lévy, s.d. (1909 selon le catalogue de la BnF); nouvelles éditions : Paris, Nelson et Calmann-Lévy, coll. « Nelson », s.d. (entre-deux-guerres) ; Paris, L'Harmattan, coll. « Autrement Mêmes », n 73, présentation de Jean-Claude Blachère, avec la collaboration de Roger Little, 2011

ONANA, Charles, René Maran, le premier Goncourt noir, 1887-1960, Paris, Duboiris, 2007 
RENAITOUR, Jean-Michel, « Après le prix Goncourt : René Maran », La Pensée française, $\mathrm{n}^{\circ} 18$ (14 janvier 1922), p. 16-18

RENAITOUR, Jean-Michel, La Bataille (15 septembre 1921)

RENAULT, Mathieu, « Amour de la race ou amour au-delà des races ? Frantz Fanon, lecteur de Maran », Présence Africaine, 187-188 ( $1^{\mathrm{er}}$ et $2^{\mathrm{e}}$ semestres, 2013), p. 231-244

RUBIALES, Lourdes, « René Maran et l'écriture du moi », dans L'Autobiographie dans l'espace francophone, II : L'Afrique, Cadiz, Servicio de publicaciones de la Universidad de Cádiz, 2005, p. 53-83.

VIOLAINES, René, « La Vie intellectuelle dans les Provinces », La Pensée française, $7^{\mathrm{e}}$ année, $\mathrm{n}^{\circ} 133$ (juillet 1927).

\section{NOTES}

1. Journal sans date, dans Euvres libres, Paris, Fayard, 1927, p. 105-236; Un homme pareil aux autres, Paris, Arc en Ciel, 1947 ; nouvelle édition : Paris, Albin Michel, 1962. Je tiens à exprimer ma vive reconnaissance à Elsa Geneste et à Lourdes Rubiales pour m'avoir aidé à établir toutes les variantes du titre au cours de nos échanges préalables au colloque Maran que nous avons coorganisé à Paris les 9 et 10 décembre 2010.

2. Voir notamment l'étude de Lourdes Rubiales, «René Maran et l'écriture du moi », dans L'Autobiographie dans l'espace francophone, II : L'Afrique, Cádiz, Servicio de publicaciones de la Universidad de Cádiz, 2005, p. 53-83. Dans sa biographie, René Maran, le premier Goncourt noir, 1887-1960, Paris, Duboiris, 2007, p. 16-18 et 45, Charles Onana cite longuement le manuscrit du Roman d'un nègre, considérant les passages comme directement autobiographiques, alors que Maran le niait résolument.

3. Information fournie par Elsa Geneste, courriel du 15 août 2010. Elsa Geneste ajoute, le 31 octobre 2010 : «Il s'agit d'une lettre du 20/12/21 [où Maran écrit] : “Je n'ai toujours pas pu ajouter une ligne ni au Petit Roi de Chimérie ni au Roman d'un nègre. Je bouillonne cependant d'idées que je voudrais fixer" qui laisse penser qu'il était déjà bien entamé ».

4. "Nous lirons de lui un autre roman significatif Le roman d'un nègre ", Jean-Michel Renaitour, «Après le prix Goncourt: René Maran », La Pensée française, n 18 (14 janvier 1922) [p. 16-18], p. 17 ; voir http://gallica.bnf.fr/ark:/12148/bpt6k58506508. Le même J.-M. Renaitour avait écrit dans La Bataille du 15 septembre 1921: «Le Batouala de René Maran vient nous convaincre définitivement qu'il est urgent que nous songions à réformer nos méthodes colonisatrices, à abdiquer nos préjugés touchant une race "inférieure", à estimer enfin qu'un homme en vaut un autre, quelle que soit la couleur de sa peau, quel que soit le climat sous lequel il est né." Cité par Ch. Onana, op. cit., p. 73, n. 9. C'est nous qui soulignons.

5. Information fournie par Elsa Geneste, courriel du 7 septembre 2010.

6. Au verso du faux-titre. Léon Bocquet en fait d'ailleurs état dans sa préface (p. 59) : «En attendant de donner ce Roman d'un nègre où l'on descendra plus avant dans l'intimité douloureuse d'une âme blessée, René Maran a parfait là-bas [...] Le Petit Roi de Chimérie que voici. » 7. Document fourni en pièce jointe par Lourdes Rubiales, courriel du 12 septembre 2010.

8. Il s'agit sans doute de la revue Eve à laquelle Maran fait allusion dans sa préface de Batouala: «Et n'est-ce pas vous, "Eve", petite curieuse, qui, au début de cette année, alors que vous étiez encore quotidienne, avez enquêté afin de savoir si une blanche pouvait épouser un nègre?» René Maran, Batouala: véritable roman nègre, édition définitive, Paris, Albin Michel, 1938 (impression de 1975), p. 11-12. 
9. Fonds privé Michel, lettre datée de Bonpasson, le 9 novembre 1925 ; information fournie par Elsa Geneste, courriel du 7 septembre 2010.

10. On sait à quel point Maran et Éboué étaient liés : voir l'introduction, par Bernard Mouralis, à la réédition de Félix Éboué, grand commis et loyal serviteur (1884-1944) de René Maran, coll. « Autrement Mêmes ", n 37, Paris, L'Harmattan, 2007.

11. Correspondance Alain Locke - René Maran, archives de Howard University, Washington D.C. ; information fournie par Elsa Geneste, courriel du 7 septembre 2010. Les titres sont soulignés d'un trait dans le texte autographe, indication usuelle au prote qu'il faut mettre l'italique.

12. René Violaines, sous la rubrique «La Vie intellectuelle dans les Provinces", La Pensée française, $7^{\mathrm{e}}$ année, $\mathrm{n}^{\circ} 133$ (juillet 1927), p. 11, $1^{\text {re }}$ col. Document fourni par Elsa Geneste en pièce jointe à son courriel du 12 septembre 2010.

13. Je songe à l'expression de Milton (Le Paradis perdu, II, 996) : « confusion worse confounded », que Chateaubriand traduit : " confusion pire que la confusion ».

14. Les Feuillets littéraires, Paris, Fayard, $n^{\circ} 4$ (1932), 39 p.

15. Cette lacune est partiellement comblée par notre étude Between Totem and Taboo: Black Man, White Woman in Francographic Literature, Exeter, University Press, 2001. Une étude préparatoire a paru sous le titre « Escaping Othello's Shadow: Un homme pareil aux autres, Ô pays, mon beau peuple! and Un chant écarlate », ASCALF Yearbook [ISSN 13653938], 1 (1996), p. 94-112.

16. D'autant que son unique contribution à La Revue du monde noir des sœurs Nardal $\left(\mathrm{n}^{\circ} 3\right.$, janvier 1932, p. 33) est le poème inédit « Othello ». Merci à Lourdes Rubiales pour cette précision. 17. Fanon consacre tout un chapitre peu tendre et peu juste à Un homme pareil aux autres dans son Peau noire, masques blancs (1952 et souvent réédité). Voir l'éclairante étude de Mathieu Renault, "Amour de la race ou amour au-delà des races? Frantz Fanon, lecteur de Maran ", Présence Africaine, 187-188 ( $1^{\text {er }}$ et $2^{\text {e }}$ semestres, 2013), p. 231-244.

18. Information fournie par Elsa Geneste, courriel du 7 septembre 2010 à partir de documents conservés par les héritiers de René Maran.

19. Un homme pareil aux autres, Paris, Albin Michel, 1962, p. 81. Il y a toutefois une évolution très nette au cours du roman. Maran écrit, p. 35, par exemple: «Un nègre n'est pas un homme comme les autres ", mais à la fin, p. 248, l'optique a changé grâce aux leçons du texte: "Vous saurez dorénavant que les nègres sont des hommes comme les autres, et qu'ils souffrent peutêtre plus que les autres, parce que, lorsqu'ils ont l'affligeant bonheur d'aimer une Européenne, ils sentent peser sur eux une telle réprobation, qu'ils ont vraiment honte d'avouer ou de laisser deviner leur souffrance. » À ma connaissance, l'étude comparative des éditions de 1947 et de 1962 n'a pas été entreprise.

20. Merci encore à Elsa Geneste pour cette information, fournie dans un courriel du 5 novembre 2010. Pour Bloch, il doit s'agir d'À la découverte du monde connu, Paris, Éditions de la NRF, 1924.

21. Cité dans notre réédition de Des inconnus chez moi, Paris, L'Harmattan, coll. «Autrement Mêmes ", n 1, 2001, p. XxIX.

22. Paris, Calmann-Lévy, s.d. (1909 selon le catalogue de la BnF); nouvelles éditions: Paris, Nelson et Calmann-Lévy, coll. «Nelson », s.d. (entre-deux-guerres) ; Paris, L'Harmattan, coll. "Autrement Mêmes", $\mathrm{n}^{\circ}$ 73, présentation de Jean-Claude Blachère, avec la collaboration de Roger Little, 2011. La pagination respective de ces deux dernières éditions est indiquée comme suit dans mon texte : (242/145).

23. C'est moi qui ajoute partout dans les citations extraites de Hiên le Maboul les italiques qui attirent l'attention sur mon thème.

24. Maran prête à Georges Lindre, dans Le Couur serré (Paris, Albin Michel, 1931, p. 47), une sensibilité qu'il semble connaître intimement: «Il n'est pas un enfant comme les autres. Il le sent, le sait et, rétractile, vit autant que possible à l'écart de ses camarades. Il les aime bien, pourtant, ses camarades [...]. » Dans sa préface au Petit Roi de Chimérie (Paris, Albin Michel, 1924, p. 13), Léon Bocquet fait remarquer la tendance chez Maran à la surcompensation : « Dès ses plus 
tendres années, afin de se venger à sa manière des avanies, il se fait un point d'honneur d'obtenir les premiers prix de grammaire. L’âge venant a développé cet orgueil: Maran a voulu se démontrer et démontrer à qui méprise les nègres qu'un Africain d'origine est aussi intelligent que n'importe quel Européen et qu'il possède parfois et écrit mieux le français que bien des autochtones du Midi ou du Nord." "Un caractère ombrageux" (ibid., p.56) ne peut que s'exaspérer face aux attaques qu'il juge injustes et déplacés, se fortifiant des leçons morales d'un stoïcisme si fortement intériorisé. « Oppose le sourire au mal, et le silence » écrit Maran dans son poème « Le visage calme » (La Vie intérieure : poèmes (1909-1912), Paris, Le Beffroi, 1912, repris dans Le Livre du souvenir : poèmes, 1909-1957, Paris, Présence Africaine, 1958).

25. Cité par Ch. Onana, op. cit., p. 22.

26. Lettre citée par J.-M. Renaitour, loc. cit., p. 18.

27. Cité par Elsa Geneste dans son étude : "Autour du Batouala de René Maran : réflexions sur quelques formulations racistes et antiracistes du mot "nègre" ", Nuevo Mundo/Nuevos Mundos, Paris, EHESS, 2010 : http://nuevomundo.revues.org/60301.

28. Un homme pareil aux autres, Paris, Albin Michel, 1962, p. 13.

29. «Vous m'avez avancé, en juin 1939, à valoir sur les droits pour un de mes romans que vous aviez projeté de publier chez vous sous le titre d'Un Nègre Parle et dont vous avez ensuite différé la publication. »

30. Lettre recueillie dans la correspondance Maran-Gahisto. La première édition de Batouala ne comporte pas d'achevé d'imprimer, juste la date 1921.

31. Dans une lettre envoyée du "Camp de Kassaï, le 4/12/1918 à 9 h ", Maran confie à Gahisto l'information suivante sur l'orthographe du chef Batouala : " avant-hier, je suis allé voir le Père Calla'ch, une lumière sur les langues d'ici. Je lui ai demandé si c'était "makoundji” qu'il fallait dire, ou bien "mokoundji" ? C'est makoundji. C'est donc "Batouala le Makoundji". Et, lorsque vous relirez l'œuvre dans son ensemble, chaque fois que vous verrez ce mot et qu'il le faudra, faites la correction nécessaire, - car j'écrivais tantôt $\underline{\text { mo }}$ tantôt ma koundji. » Maran n'a enfin retenu que Batouala pour les versions de 1921 et de 1938 du roman.

\section{RÉSUMÉS}

René Maran revient à plusieurs reprises sur le titre du roman qui, dans son édition définitive, s'appellera Un homme pareil aux autres. Longtemps, il portait le titre Le Roman d'un Nègre et cette étude, publiée d'abord en 2013 dans la revue Présence africaine, retrace les variations à partir des débuts de ce projet, en 1920, jusqu'à la version définitive, tout en y ajoutant en post-scriptum une mise à jour qui tient compte des manuscrits devenus accessibles depuis.

René Maran has several changes of mind over the title to be given to the novel which will eventually be published as Un homme pareil aux autres. For a considerable time, it was called Le Roman d'un Nègre and this study, first published in 2013 in the periodical Présence africaine, retraces the variations from its earliest mention in 1920 to the final version, adding in a postscript an update taking account of newly available manuscripts. 
INDEX

Mots-clés : René Maran, Variations sur un titre, Roman d'un Nègre, Journal sans date, Un homme pareil aux autres, Émile Nolly, Hiên le Maboul

Keywords : René Maran, Variations on a title, Roman d'un Nègre, Journal sans date, Un homme pareil aux autres, Émile Nolly, Hiên le Maboul

\section{AUTEUR}

\section{ROGER LITTLE}

Ancien titulaire de la chaire de français du Trinity College Dublin. 